\title{
Analytical and Non - analytical Scatterers in Plane Waveguide with Hard Elastic Bottom, Irradiated by Pulse Sound Signal
}

\author{
Alexander Kleshchev \\ Department of Physics, Saint-Petersburg State Navy Technical University, Saint-Petersburg, Russia \\ Email address: \\ alexalex-2@yandex.ru \\ To cite this article: \\ Alexander Kleshchev. Analytical and Non - analytical Scatterers in Plane Waveguide with Hard Elastic Bottom, Irradiated by Pulse Sound \\ Signal. American Journal of Modern Physics. Vol. 6, No. 4, 2017, pp. 51-55. doi: 10.11648/j.ajmp.20170604.12
}

Received: May 19, 2017; Accepted: June 1, 2017; Published: July 7, 2017

\begin{abstract}
Based on the method of imagenary sources and imagenary scatterers is the solution of the problem of the sound diffraction by pulse signals at ideal (soft) prolate spheroid, put in the plane waveguide with the hard elastic bottom. In the work is proved that with such a formulation of problems eliminated possibility of using the method of normal waves because pulses are bundies of energy and can therefore only be distributed to the group velocity which is inherent in just the method of imaginary sources. Calculations made in the article shoved that imagenary sources with smail numbers experienci8ng the effect of total internal reflection, as the result of the reflection coefficient $V$ by the hard elastic bottom is complex and the real part of $V$ is close to 1,0 which corresponds $V$ absolutely hard bottom. Found sequences of reflected pulses for the elastic hard bottom and the absolutely hard bottom floor confirmed this approach. In the final part of the arti8cle on the basis of the received results given by a solution (the method integral equations) is much more complex problem of the diffraction at the elastic non-analytical scatterer, put in the plane waveguide witch the hard elastic bottom.
\end{abstract}

Keywords: Scatterer, Prolate Spheroid, Imaginary Source, Diffraction, Elastic Hard Bottom, Boundary Conditions, Group Velocity, Phase Velocity

\section{Introduction}

Will it is known [1] thay the sound signal as a bundle of energy propagates with the group velocity. This fact forces us to use method of imaginary sources in the study of the temporal characteristics of pulse signals scattered by various bodies put in plane wavegu-ide [2 - 7]. While spectral characteristics of dealing with continuous harmonic signals can be investigated using the method of normal waves [8]. In previous syudies of the sound field in plane waveguides either a liquid or absolutely hard bottom was considered, in this article is first studied the waveguide with a hard elastic bottom.

\section{Diffraction Pulse Sound Signal on the Soft Prolate Spheroid Located in Plane Waveguide with the Hard Elastic Bottom}

We turn to a familiar problem of the diffraction of pulses on spheroial bodies I the pla-ne waveguide [2 - 4, 7], retairing the upper boundary condition Dirichlet, waveguide di-mensions and scatterer with respect to boundaries, replasing only ideal hard lower bounda-ry on the elastic isotropic bottom. Physical parameters of the lower medium will corres-pond to the isotropic elastic bottom, but in their values, they will be very close to parame-ters of transverselyisotropic rock - a large gray siltstone [9]. The longitudinal wave velocity in this material is $4750 \mathrm{~m} / \mathrm{s}$, the transverse wave velocity $-2811 \mathrm{~m} / \mathrm{s}$. When used in this case the method of imaginary sources need to enter the reflection coefficient $V$ 
for the each source [10], when displaying sources relative to the upper border sources, as before [2 - 7], will change the sign on the opposite, which corresponds to a change of phase by $\pi$

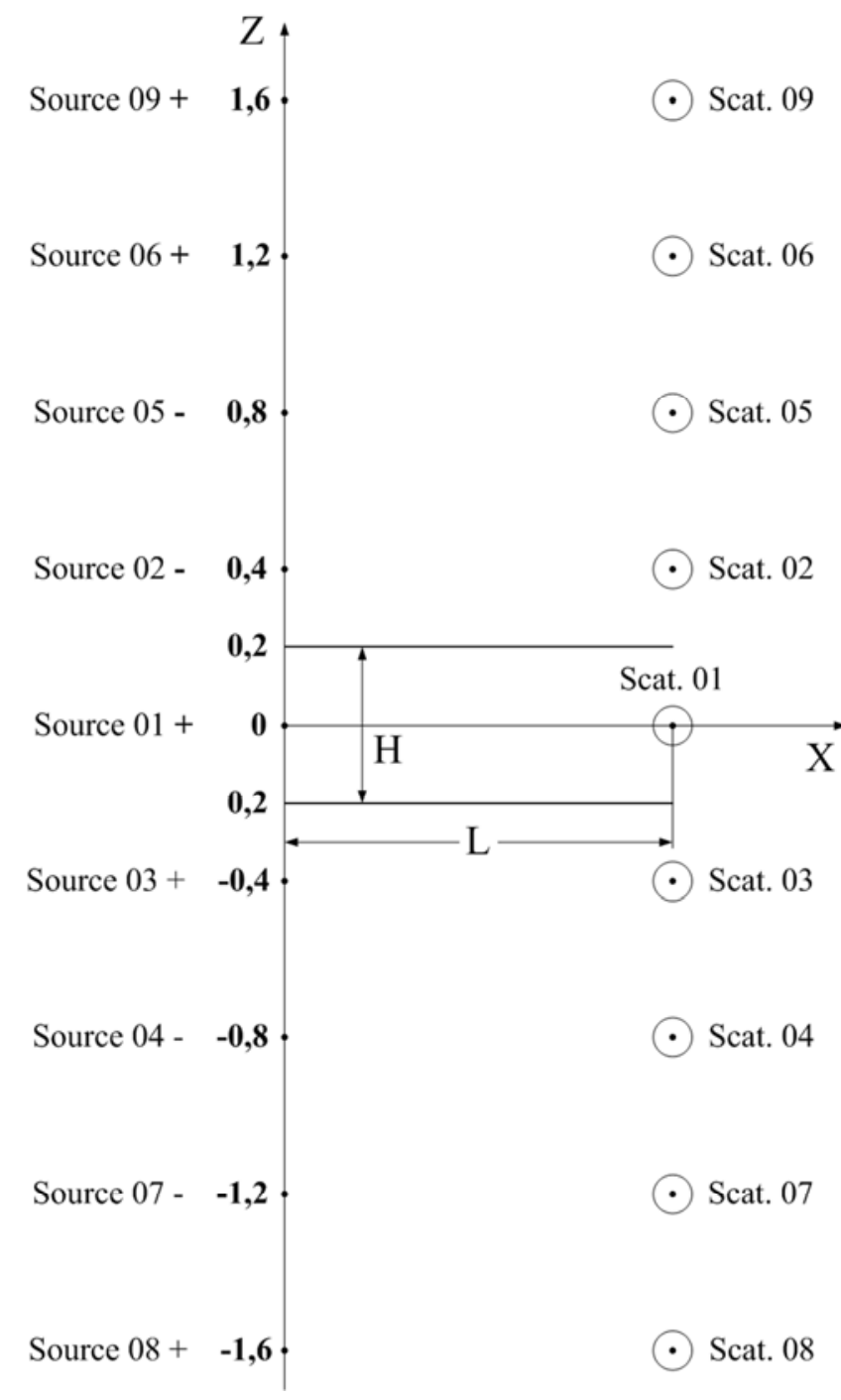

Figure 1. The mutual disposition of the pulse point-soirces and scatterers in the plane waveguide.

It is known to [10], that the of imaginary sources method boundary conditions are not fulfilled strictly on any of borders of the waveguide even in the case of ideal boundary conditions of Dirichlet and Neumann. For the better fulfillment of these conditions in diffraction problems $[2-7$, 11] were introduced imaginary scatterers by mirroring their relatively waveguide boundaries. Likewise conduct imaginary scatterers and in our prob-lem and compare the sequence of reflected pulses $[2,3,11]$ in the case of ideal borders and in presence of a hard elastic bottom in the waveguide. In [10] shows that the method of imaginary sources applicable in the case where the reflection coefficient $V$ will be a func-tion of the angle of the incidence of the wave from a source relati-ve to the normal to the boundary. In our case this angle will be determined by the mutual position of the source (real or imaginary) and the scatterers (real or imaginary), which falls the wave from the so-urce. Since the receiver is combined with a real source $Q$, the sequence of reflected pulses will be determined by the quantity and amplitudes of reflected signals (from different scat-terers) having the same propagation time from sources to scatterers and from scatterers to the point $Q$. Parameters of the waveguide, the position of the real source $Q$ (combined with the receiver) and the real scatterer remained unchanged compared [2, 3, 11]: $L=1000 \mathrm{~m}$., $H=400 \mathrm{~m}$., the real source $Q$ and real scatterer are located at the depth of $200 \mathrm{~m}$., the scatterer is the ideal soft prolate spheroid with the semi-axes ratio $a / b=10(a=0,279 \mathrm{~m}$.) and its axis of a rotation is perpendicular to the plane of the figure (see Figure 1). The formula for the reflection coefficient $V_{0 N}$, where $N$ - the number of a source, is given in [10]. For the calculatio9nof first five of reflected pulses us needed following reflection coefficients: $V_{03}$ in the direction of the first (real) scatterer $01, V_{05}$ in the direction of the second (imaginary) scatterer 02, $V_{06}$ in the direction of same the second scatterer.

As a result of simple calculations with the help of [10] obtain: $V_{03}=0,9989+i 0,0633$;

$$
V_{05}=0,9989+i 0,0633 ; V_{06}=0,6238+i 0,7897 \text {. All }
$$

three coefficients have turned complex, which mesns the total internal reflection at the boundary liquid - hard elastic bottom, therefore all three modules of reflection coefficients are equal 1,0 and real parts of first two coefficients are close to $+1,0$, which is typical for the boundary liquid - absolutely hard bottom. The resulting sequence of calculations of first five reflected pulses is shown in Figure 2. We compare them to the sequence in figure 3 for ideal boundaries $[2,3,11]: 1^{\text {st }}$ and $4^{\text {th }}$ pulsews of Figure. 2 are identical with first and second pulses of Figure 3, as for for $2^{\text {nd }}, 3^{\text {rd }}$ and $5^{\text {th }}$ pulses in Figure 2 in the case of ideal boundaries and symmetrical location of real a source and a scatterer relatively of boundaries of the waveguide, they are compensated each reflected pulses, i. e. $2^{\text {nd }}, 3^{\text {rd }}$ and $5^{\text {th }}$ pulses (see Figure. 2) show the difference in se-quences of reflected pulses when replacing an absolutely hard bottom on an elastic hard bottom.

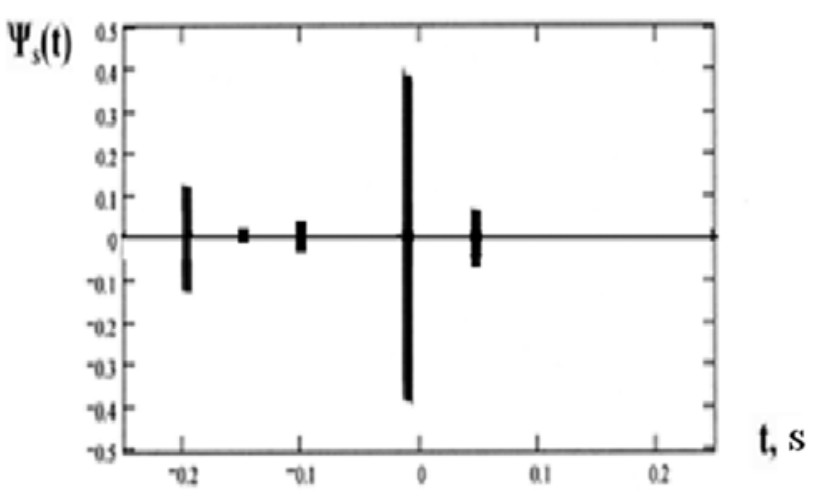

Figure 2. The normalized series of first five reflected impulses in the waveguide with the hard elastic bottom.

A similar pattern is observed for anisotropic bottom, such as silicon, in wich the velocity of the quasi-longitudinal wave of about $8300 \mathrm{~m} / \mathrm{s}$ and quasi-transverse wave velocity of 
about $5700 \mathrm{~m} / \mathrm{s}$, with the secjnd quasi-transverse wave do not occur because of the problem statement [9]. Because of the high velocities of quasi - longitudinal and quasi - transverse waves total internal reflection effect at the anisotropic bottom manifest itself even more strongly than the isotrohic bottom.

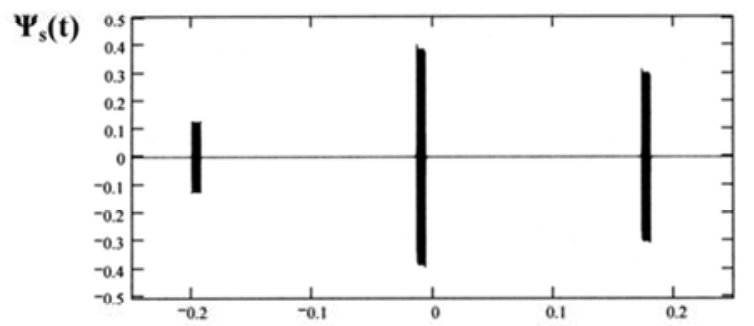

t, $\mathrm{s}$

Figure 3. The normalized series of first three reflected impulses with the harmonic filling in the point $Q$.

\section{Diffraction Pulse Sound Signal on Non-analytical Scatterer Put in Plane Waveguide with Hard Elastic Bottom}

Based on the obtained solution, consider a more general problem of the diffraction of the pulsed sound signal on an elastic scatterer as a finite cylindrical shell, supplemented with two hemispherical shells (figure 4) and placed in the waveguide with an elastic hard bottom (figure 5), using the method of integral equations $[11-16]$. We note that a similar problem can be solved with the help of other methods: the boundary element method $[15,17] ; \mathrm{T}$ - matrix method [18]; the method of a potential [19]; the finite element method [20]; the method of Green's functions [21].

The first stage will solve the problem of the diffraction of a harmonic wave on a such shell.

The density of the material of the shell is $\rho_{l}$, the Lame's coefficients $-\lambda$ and $\mu$. The shell was filled in the internal liquid medium with the density $\rho_{2}$ and the sound velocity $C_{3}$ and it was placad in the external liquid medium with the density $\rho_{0}$ and the sound velocity $C_{0}$. At the shell falls the plane harmonic wave with pressure $p_{i}$ under the angle $\Theta_{0}$ and with the wave vector $\vec{k}$

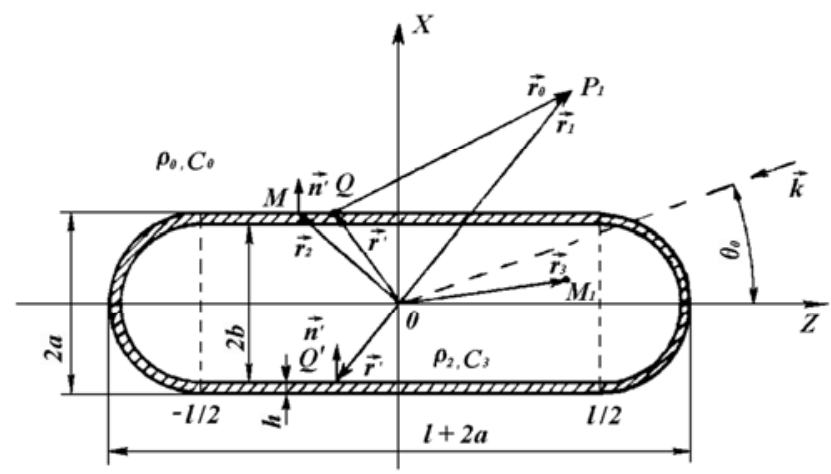

Figure 4. The elastic shell in the form of the terminal cylinder with the semi - spheres.

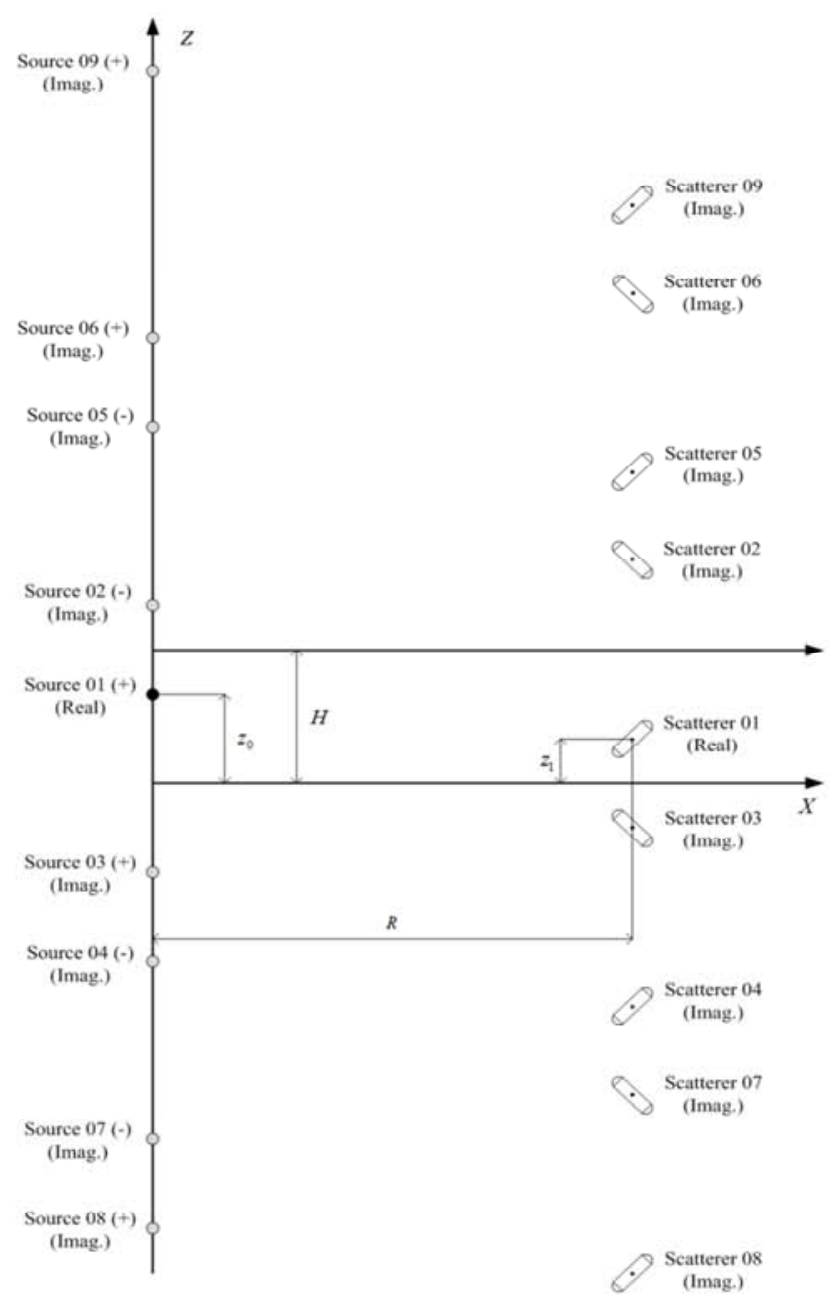

Figure 5. The mutual disposition of the impulse point-sources and scatterers in the plane waweguide.

As was shown in [11 - 16], the initial equation is integral equation, having the sense of the generalized Huygen's principle, for the displacement vector $\vec{u}(\vec{r})$ of the elastic shell:

$$
\vec{u}(\vec{r})=\iint_{S}\left\{\vec{t}\left(\vec{r}^{\prime}\right) G\left(\vec{r}^{\prime} ; \vec{r}\right)-\vec{u}\left(\vec{r}^{\prime}\right)\left[\hat{n}^{\prime} \sum\left(\vec{r}^{\prime} ; \vec{r}\right)\right]\right\} d S\left(\vec{r}^{\prime}\right), \quad \vec{r} \in V
$$

where $\vec{t}\left(\vec{r}^{\prime}\right)=\hat{n}^{\prime} T\left(\vec{r}^{\prime}\right) \quad$ is the stress vector; $\hat{n}^{\prime} \equiv \hat{n}^{\prime}\left(\vec{r}^{\prime}\right)=\vec{n}^{\prime}\left(\vec{r}^{\prime}\right)$ is the single vector of the external along the relation to $S$ normal; $T\left(\vec{r}^{\prime}\right)$ is the stress tensor of the isotropic material; $G\left(\vec{r}^{\prime} ; \vec{r}\right)$ is the displacement Green's tensor; $\sum\left(\vec{r}^{\prime} ; \vec{r}\right)$ is the stress Green's tensor; if $\vec{r}$ concerns to the point of the surface $S$, in the left part of the equation (12) will stand $\vec{u}\left(\vec{r}^{\prime}\right) / 2$.

The displacement vector $\vec{u}(\vec{r})$, the stress tensor $T(\vec{r})$, the displacement Green's tensor

The displacement vector $\vec{u}(\vec{r})$, the stress tensor $T(\vec{r})$, the displacement Green's tensor $G\left(\vec{r}^{\prime} ; \vec{r}\right)$ and the stress Green's 
tensor $\Sigma\left(\vec{r}^{\prime} ; \vec{r}\right)$ were connected between by the following correlations $[11,16]$ :

$$
T(\vec{r})=\lambda \operatorname{I} \nabla \vec{u}(\vec{r})+\mu(\nabla \vec{u}+\vec{u} \nabla),
$$

where $\quad \mathrm{I}=\mathrm{I}_{L}+\mathrm{I}_{T} \quad ; \quad \mathrm{I}_{L}=(\nabla \nabla) / \nabla^{2} \quad ; \quad \mathrm{I}_{L} \cdot \mathrm{I}_{T}=0 \quad ;$ $\mathrm{I}_{T}=-[\nabla(\nabla \mathrm{I})] / \nabla^{2}, \mathrm{I}_{L}$ and $\mathrm{I}_{T}$ are the longitudinal and transverse single tensors for the Hamilton's operator $\nabla$;

$$
\begin{gathered}
\sum\left(\vec{r}^{\prime} ; \vec{r}\right)=\lambda \operatorname{I} \nabla G\left(\vec{r}^{\prime} ; \vec{r}\right)+\mu\left[\nabla G\left(\vec{r}^{\prime} ; \vec{r}\right)+G\left(\vec{r}^{\prime} ; \vec{r}\right) \nabla\right] ; \\
G\left(\vec{r}^{\prime} ; \vec{r}\right)=\left(1 / 4 \pi \rho_{t} \omega^{2}\right)\left\{k_{2} \operatorname{I} g\left(k_{2}\left|\vec{r}^{\prime}-\vec{r}\right|\right)+\right. \\
\left.+\nabla^{\prime}\left[g\left(k_{1}\left|\vec{r}^{\prime}-\vec{r}\right|\right)-g\left(k_{2}\left|\vec{r}^{\prime}-\vec{r}\right|\right)\right] \nabla\right\},
\end{gathered}
$$

where $k_{1}$ and $k_{2}$ are the wave numbers of the longitudinal and transwerse waves in the material of the shell; $g\left(k_{2}\left|\vec{r}^{\prime}-\vec{r}\right|\right)=\exp \left(i k_{2}\left|\vec{r}^{\prime}-\vec{r}\right|\right) / 4 \pi\left|\vec{r}^{\prime}-\vec{r}\right| \quad$ is the Green's function.

The second integral equation presents the Kirchhoff integral for the diffracted pressure $p_{\Sigma}\left(P_{1}\right)$ in the external medium:

$$
\begin{gathered}
C\left(P_{1}\right) p_{\Sigma}\left(P_{1}\right)=-\iint_{S_{a}}\left\{p_{\Sigma}(Q)\left(\partial / \partial n^{\prime}\right)\left[\exp \left(i k r_{0} / r_{0}\right)\right]-\right. \\
\left.-\left[\exp \left(i k r_{0} / r_{0}\right)\right] \rho_{0} \omega^{2}\left(\vec{u} \vec{n}^{\prime}\right)\right\} d S_{a}+4 \pi p_{i}\left(P_{1}\right),
\end{gathered}
$$

where $p_{\Sigma}\left(P_{1}\right)=p_{i}\left(P_{1}\right)+p_{s}\left(P_{1}\right) ; p_{s}\left(P_{1}\right)$ is the scattered pressure in the point $P_{l} ; C\left(P_{l}\right)$ is the numerical coefficient, equal $2 \pi$, if $P_{1} \in S_{a}$ and $4 \pi$, if $P_{l}$ out $S_{a} ; S_{a}$ is the external surface of the shell; $Q$ is the point of the external surface of the shell.

For the pressure $p_{2}\left(M_{1}\right)$ in the internal liquid medium in the point $M_{l}$ is got the third integral equation:

$$
\begin{gathered}
C\left(M_{1}\right) p_{2}\left(M_{1}\right)=\iint_{S_{b}}\left\{p_{2}\left(Q^{\prime}\right)\left(\partial / \partial n^{\prime}\right)\left[\exp \left(i k r_{3}\right) / r_{3}\right]-\right. \\
\left.-\left[\exp \left(i k r_{3}\right) / r_{3}\right] \rho_{0} \omega^{2}\left(\vec{u} \vec{n}^{\prime}\right)\right\} d S_{b},
\end{gathered}
$$

where $Q^{\prime}$ is the point of the inte;rnal surface of the shell;

$$
C\left(M_{1}\right)=\left\{\begin{array}{lll}
4 \pi, & \text { if } & M_{1} \text { out } S_{b} \\
2 \pi & \text { if } & M_{1} \in S_{b}
\end{array}\right.
$$

$S_{b}$ is the internal surface of the shell.

To the integral equations (1), (5) and (6) are added the boundary conditions on the external $\left(S_{a}\right)$ and internal $\left(S_{b}\right)$ surfaces of the shell:

1 - at the both surfaces of the shell the tangent stresses are equally null:

$$
\left.\tau_{i}\right|_{S_{a}}=0 ;\left.\quad \tau_{i}\right|_{S_{b}}=0 ; \quad i=1,2
$$

2 - the normal stress $\sigma_{n^{\prime}}$ at the external surface of the shell is equally the diffracted pressure $p_{\Sigma}$, but at the internal surface is equally the pressure $p_{2}$

$$
\left.\sigma_{n^{\prime}}\right|_{S_{a}}=p_{\Sigma} ;\left.\quad \sigma_{n^{\prime}}\right|_{S_{b}}=p_{2}
$$

In the conformity with the conditions (7) and (8) the stress vector $\vec{t}\left(\vec{r}^{\prime}\right)$ in the equation (1) is equal:

$$
\vec{t}\left(\vec{r}^{\prime}\right)=\left.p_{\Sigma} \vec{n}^{\prime}\right|_{S_{a}} ; \quad \vec{t}\left(\vec{r}^{\prime}\right)=\left.p_{2} \vec{n}^{\prime}\right|_{S_{b}} ;
$$

3 - the continuity of the normal component of the displacement at the both boundaries of the shell:

$$
\left.\begin{array}{l}
u_{n^{\prime}}=\left.\left(1 / \rho_{0} \omega^{2}\right)\left(\partial p_{\Sigma} / \partial n^{\prime}\right)\right|_{S_{a}} ; \\
u_{n^{\prime}}=\left.\left(1 / \rho_{2} \omega^{2}\right)\left(\partial p_{2} / \partial n^{\prime}\right)\right|_{S_{b}} \cdot
\end{array}\right\}
$$

The substitution of the integral equations (6), (1) and (7) in the boundary conditions (18) - (20) gives the system of equations in terms of unknown functions $p_{\Sigma}, p_{2}$ and the components of the displacement vector $\vec{u}$ at the both surfaces of the shell. To obtain numerical solution of this system the integral equations are replaced the quadrature formulas and the grid of the nodal points is chosen at both surfaces of the shell as well as it has be done for the ideal non - analytical scatterers [ 11,12$]$.

For choosing boundary conditions we will have the integrals of the two types: the integrals with the isolated special point and the integrals which are considered of the sence of the principal meaning. The method of the calculation of the second types was described in [11].

Thus calculated reflection characteristics of the harmonic signal with frequency $v$ can determine the spectral reflectance function $S_{S}(2 \pi v)$ and it can help be applying a Fourier transform we obtain a temporary function of the reflected pulse $\Psi_{S}\left(t^{\prime}\right)[22]$ :

$$
\Psi_{S}\left(t^{\prime}\right)=\frac{1}{\pi} \operatorname{Re} \int_{0}^{\infty} S_{S}(2 \pi v) e^{+i 2 \pi v t} d(2 \pi v)
$$

Similarly using spectral reflectance characteristics of elastic bodies of spheroidal form [11, 23 - 26], we can compute sequences of reflected pulses in the waveguide with hard elastic bottom and for their.

\section{Conclusions}

As a result of the research we can draw three conclusions:

1) in studying propagation and diffraction of pulse signals in a plane waveguide need to use the method of imaginary sources as pulses like bundles of energy spread to any direc-tions (including and along the axis of the waveguide) with the group velocity does not exceed the sound velocity, namely the group velocity 
based the method of imaginary sources;

2) replacing the hard elastic bottom on the absolutely hard bottom is acceptable to those sources (real and imaginary) from which waves in the fall to the hard elastic bottom try total internal reflectionж

3 ) we have adopted the model of image sources and image scatterers is gute acceptable (due to internal reflection), at least, for first five calculated reflected pulses in a plane wave-guide with hard elastic bottom.

\section{References}

[1] Kolskiy G. Waves of Stresses in Hard Bodies. M.: I L, 1956. $272 \mathrm{p}$.

[2] Kleshchev A. A.,Kuznetsova E. I. Scattering of pulse sound signals by the spheroidal body, put in plane wavegui de. $/$ Coll. Proc. Russ. Acoust. Soc. XXIV session. M.: GEOS. 2011. V. 1. P. 743-745.

[3] Kleshchev A. A., Kuznetsova E. I. Diiffraction of Impulse Signals on Spheroidal Body, Put in Plane Waveguide/ / International Journal of Theoretical and Mathematical Physics. 2012. V. 2. № 6. P. 211-214.

[4] Kleshchev A. A. Diffraction of Pulse Sound Signals on Elastic Spheroidal Shell, Put in Plane Waveguide. / Advanced Studies in Theoretical Physics. 2013. V. 7. № 13 - 16/ P. 697-705.

[5] Kleshchev A. A, Diffraction of Sound Signals at Elastic Shell of Non-analytical Form Put in Plane Waveguide. / Advances in Signal Processing. 2014. V. 2. № 2. P. 46-49.

[6] Kleshchev A. A. Pulse Sound Signals Diffraction on Elastic Bodies of Analytical and Nonana-lytical Forms, Put in Plane Waveguide. / Zeitschrift fur Naturforschung A. 2015. V. 70. № 6. P. 419-427.

[7] Kleshchev A. A. Diffraction of Pulse Sound Signals on Elastic Bodies of Spheroidal Form Put in Plane Waveguide// MIT. 2015. V. 2. № 28. P. 77-81.

[8] Kleshchev A. A., Klyukin I. I. The spectral characteristics of the scattering of the sound by body, placed in the sound channel. / Sov. Phys. Acoust. 1974. V. 20. № 3. P. 470 - 473.

[9] Lekhnitskiei S. G. Theory of Elasticity of Anisotropic Elastic Body. M.: Science, 1977. 416 p.

[10] Brechovakikh L. M. Waves in Laminated Mediums. M.: Publ. Acad. Scien. SSSR, 1957. 502 p.

[11] Kleshchev A. A. Hydroacoustic Scatterers. S.-Pb.: Prima, 2012. $268 \mathrm{p}$.
[12] Kleshchev A. A. Scattering of Sound by Ideal Bodies of Nonanalytical Form. / Tr. Lenin. Korablestr. Inst. 1989. Generalship. Syst. P. 95-99.

[13] Kleshchev A. A. Method of Integral Equations in Problem of Sound Diffraction on Elastic Shell of Non-analytical Form. / Techn. Acoust. 1993. V. 2. № 4(6). P. 65-66..

[14] Kleshchev A. A. Method of Integral Equations in Problem of Sound Diffraction on Bodies of Non-analytical Form. / Marine messenger. 2013. № 2(125). P. 94-98.

[15] Seybert A. F., Wu T. w, and Wu X. F. Radiation and scattering of acoustic waves from elas-tic solids and shells using the boundary element method. / J. A. S. A. 1988. V. 84. № 5. P. 1906-1912.

[16] Podstrigach J. S., Poddubnjak. Scattering of Sound Beams of Spherical and Cylindrical Form. Kiev: Naukova Dumka, 1986. $264 \mathrm{p}$.

[17] Brebbia C. A., Walker S. Boundary Element Techniques in Engineering. M.: Mir, 1982. 242 p.

[18] Peterson B., Strom S. Matrix Formulation of Acoustic Scattering from Multilayered Scatte-rers. / J. A. S. A. 1975. V/ 57. № 1. P. 2-13.

[19] Kupradze V. D. Methods of Potential in Theory of Elasticity. M.: Fizmatgiz, 1963. 472 p.

[20] Dushin A. Yu., Il'menkov S. L., Kleshchev A. A., Postnov V. A. Use of Finite El Sement Method to Solution of Problems of Sound Radiating by Elastic Shells. / Proc. All-Union Symp. Interaction of Acoustical Waves with Elastic Bodies. Tallinn: 1989. P. 89-91.

[21] Il'menkov S. L., Kleshchev A. A., Klimenkov A. S. The Green's Function Method in the Problem of Sound Diffraction by an Elastic Shell of Noncanonical Shape. / Acoust. Phys. 2014. V. 60. № 6. P. 579-586.

[22] Kharcevich A. A. Spectrum and Analisis. M.: GITTL, 1957. $236 \mathrm{p}$.

[23] Kleshchev A. A. Three-Dimensional and Two-Dimensional (Axis-Symmetrical Characteris-tics of Elastic Spheroidal Scatterers. / Akust. Zh. 1986. V. 32. № 2. P. 268-270.

[24] Kleshchev A. A., Klyukin I. I. Principles of Hydroacoustics. L.: Shipbuilding, 1987, 224 p.

[25] Kleshchev A. A. Debye and Debye-Type Potentials in Diffraction, Radiation and Elastic Wave Propagation Problems. / Acoust. Phys. 2012. V. 58. № 3. P. 338-341.

[26] Kleshchev A. A. Resonance Scattering of Sound by Spheroidal Elastic Bodies and Shells. / Acoust. Phys. 2014. V. 60. № 3. P. $253-261$. 